\title{
Electronic Band Structure and Superconducting Properties of SnAs
}

\author{
P. I. Bezotosnyi,, औ K. A. Dmitrieva,${ }^{1}$ A. V. Sadakov, ${ }^{1}$ K. S. Pervakov, ${ }^{1}$ \\ A. V. Muratov, ${ }^{1}$ A. S. Usoltsev, ${ }^{1,2}$ A. Yu. Tsvetkov, ${ }^{1}$ S. Yu. Gavrilkin, ${ }^{1}$ N. S. Pavlov, ${ }^{3}$ \\ A. A. Slobodchikov ${ }^{3,4}$ O. Yu. Vilkov, ${ }^{5}$ A. G. Rybkin, ${ }^{5}$ I. A. Nekrasov ${ }^{3}$ and V. M. Pudalov ${ }^{1,2}$ \\ ${ }^{1}$ P. N. Lebedev Physical Institute, Russian Academy of Sciences, Moscow, 119991, Russia \\ ${ }^{2}$ National Research University Higher School of Economics, Moscow 101000, Russia \\ ${ }^{3}$ Institute for Electrophysics, Russian Academy of Sciences, Ural Branch, Ekaterinburg, 620016, Russia \\ ${ }^{4}$ Kirensky Institute of Physics, Federal Research Center KSC SB RAS, Krasnoyarsk, 660036, Russia \\ ${ }^{5}$ Saint Petersburg State University, Saint Petersburg, 198504, Russia
}

\begin{abstract}
We report comprehensive study of physical properties of the binary superconductor compound SnAs. The electronic band structure of SnAs was investigated using both angle-resolved photoemission spectroscopy (ARPES) in a wide binding energy range and density functional theory (DFT) within generalized gradient approximation (GGA). The DFT/GGA calculations were done including spin-orbit coupling for both bulk and (111) slab crystal structures. Comparison of the DFT/GGA band dispersions with ARPES data shows that (111) slab much better describes ARPES data than just bulk bands. Superconducting properties of SnAs were studied experimentally by specific heat, magnetic susceptibility, magnetotransport measurements and Andreev reflection spectroscopy. Temperature dependences of the superconducting gap and of the specific heat were found to be well consistent with those expected for the single band BCS superconductors with an isotropic s-wave order parameter. Despite spin-orbit coupling is present in SnAs, our data shows no signatures of a potential unconventional superconductivity, and the characteristic BCS ratio $2 \Delta / T_{c}=3.48-3.73$ is very close to the BCS value in the weak coupling limit.
\end{abstract}

\section{INTRODUCTION}

Binary compounds of the SnX-family $(\mathrm{X}=\mathrm{Te}, \mathrm{Se}, \mathrm{As}$, $\mathrm{S}, \mathrm{Sb}, \mathrm{P}$ ) attract much attention due to their unique properties [1 5]. The most extensively studied SnSe [6] and SnTe [7, 8] have NaCl-structure. These compounds were theoretically and experimentally determined as topological crystalline insulators. In this class of materials topological properties are protected by crystal symmetries, that differs from conventional topological insulators, where the time-reversal symmetry is the determining factor [9]. Moreover, In-doped SnTe was found to manifest signatures of Andreev bound states [10], which are characteristic of unconventional superconductivity. Given this fact and the topological nature of the material, $\mathrm{Sn}_{1-x} \operatorname{In}_{x}$ Te is considered as a strong candidate for a topological superconductor [11, 12].

Tin arsenide is isostructural to SnTe and SnSe. Without doping, this compound demonstrates superconducting properties, which were first reported in 1964 by Geller and Hull [13]. For a long time there was an uncertainty about valence state of $\mathrm{Sn}$ in this compound and about its possible influence on superconductivity. Later, Wang et. al 14] experimentally demonstrated that $\mathrm{Sn}$ has a single valence state $\mathrm{Sn}^{+3}$. Also, from rough estimates based on experimental data it was suggested, that $\mathrm{SnAs}$ is a type-I superconductor with weak-coupling. Temperature dependences of the critical magnetic field $H_{\mathrm{c}}$ and of the specific heat in zero field are well described by the BCS model. Nevertheless, up to the best of our knowledge no information is available on the specific heat behavior in non-zero magnetic fields and on the temperature depen- dence of the superconducting gap. Furthermore, from earlier theoretical investigation of electronic band structure, electron-phonon interaction, and superconductivity performed in 15], the calculated electron-phonon coupling parameter seemed to agree with the one estimated from specific heat data [14], however there is a discrepancy between the theoretical and experimental values of $\mathrm{T}_{c}$.

Another interesting result was found in investigations under high-pressure. In Ref. 16 SnAs was shown to exhibit a structural phase transition from $\mathrm{NaCl}$ - to $\mathrm{CsCl}-$ structure at around $37 \mathrm{GPa}$, which confirms previously obtained experimental results [17]. Moreover, there is dramatic increase of $T_{\mathrm{c}}$ up to $12.2 \mathrm{~K}$ at a critical point of the structural transition.

On the theoretical side, in Ref. [18] electronic band structure of SnAs was calculated within the tightbinding approximation. Despite the experimental data of Ref. 14 demonstrated that $\mathrm{Sn}$ has a single valence state $\mathrm{Sn}^{+3}$, this compound is considered [18] as a potential candidate for the valence-skip material. The valence skipping may lead to a negative effective Coulomb interaction between electrons. The calculation results reveal that the $\mathrm{Sn}^{x}$ state in SnAs is likely intermediate between valence skipper and fixed valence compound; as a result, moderate charge fluctuations (and electron-phonon interaction) can be responsible for superconductivity.

The band dispersion of SnAs resembles that for SnTe, the known crystalline topological insulator. Moreover, due to the similarity of SnAs to SnTe, it was suggested, that the mixed compound $\mathrm{Sn}(\mathrm{As}, \mathrm{Te})$ may manifest topological superconductivity, if it is superconducting. Pre- 
liminary experimental studies of electronic band structure of SnAs by ARPES technique [19] have shown the presence of features that can be interpreted as a manifestation of the spin-orbit coupling (SOC): energy band splitting in the vicinity of the $\Gamma$ point, which might be important in context of possible topological nature of the mixed compound $\mathrm{Sn}(\mathrm{As}, \mathrm{Te})$. The ARPES data [19] was obtained for the (111) sample surface, whereas band structure calculations are available for bulk dispersion solely. This impedes comparison of the data with theory; obviously, one needs band structure calculated within the corresponding cross-section of the Brillouin zone.

Despite the existence of previous investigations, there is still lack of knowledge of the origin of superconductivity in SnAs, and the question about the impact of SOC is still opened. In the current paper we report detailed experimental study of the normal and superconducting properties of SnAs. For the normal state we studied by ARPES technique electronic band structure in a wide binding energy interval. The measured superconducting properties include specific heat and magnetic susceptibility in various magnetic felds, and the superconducting gap by Andreev reflection spectroscopy. Also, we report refined calculations of the SnAs band structure within DFT/GGA for bulk and (111) slab crystal structures, with and without SOC, and compare them with the ARPES spectra.

\section{SAMPLES AND EXPERIMENTAL DETAILS}
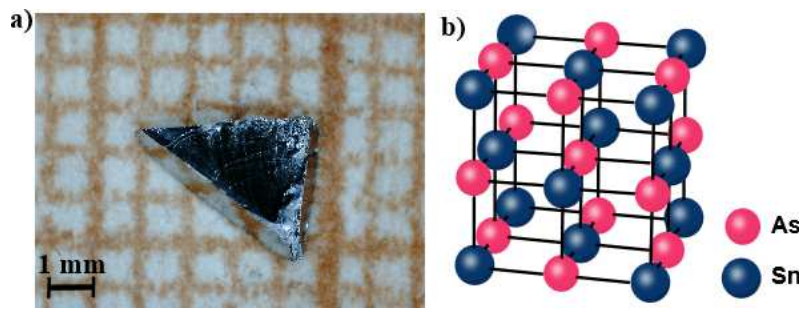

Figure 1. a) Image of the SnAs crystal. b) Crystal structure of SnAs.

The SnAs samples were synthesized from pure elements: Sn and As with purity of $99.99 \%$ and $99.9999 \%$, respectively. The sealed quartz ampoule with elements taken in a stoichiometric ratio of 1:1 was slowly heated to $600^{\circ} \mathrm{C}$, held for 48 hours to form the phase, then heated to $800^{\circ} \mathrm{C}$ and held for 24 hours to homogenize the melt. The crystals were then grown by a modified Bridgman method at a melt cooling rate of $1^{\circ} \mathrm{C} /$ hour down to $550^{\circ} \mathrm{C}$. The grown crystals were further annealed at a temperature of $550^{\circ} \mathrm{C}$ for 24 hours to remove growth defects.

We studied two crystals (No.1 and No.2) obtained in two different growths. Being nominally identical, the

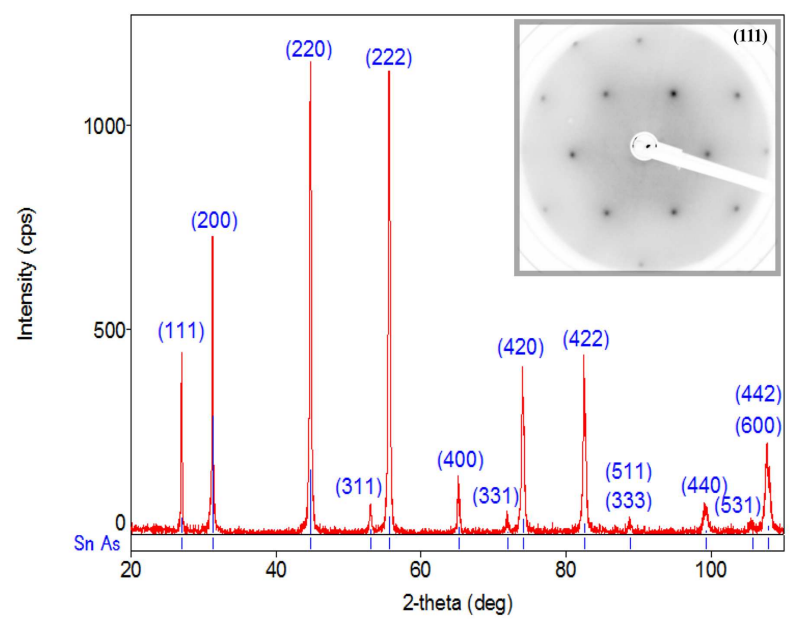

Figure 2. XRD spectrum of the SnAs (sample No. 2). The abscissa represents the angle $2 \Theta$ in degrees, the ordinate is the reflection intensity. Inset: LEED pattern for SnAs crystal with electron beam energy $E=120 \mathrm{eV}$.

samples however had slightly different $T_{c}$ values. For ARPES measurements both SnAs samples were used, for resistivity and Andreev spectroscopy we used sample No. 1 , for specific heat and magnetic measurements - sample No. 2.

The obtained SnAs crystals were cleaved into the small pieces of about $3 \mathrm{~mm}$ size, which had a pyramidal shape with triangular base (Fig. 1(a)). Structural investigations of both SnAs crystals were done with Rigaku MiniFlex 600 X-ray powder diffractometer. The measured diffraction pattern of SnAs is shown in Fig. 2, It contains only reflexes related to the SnAs phase, and no reflections intrinsic to other phases. From XRD analysis we confirmed the NaCl-type structure (Fig. T(b)) and found the lattice parameter $a=5.723 \AA$ to be in agreement with earlier reported data [14]. The quality of the sample surface was investigated in situ by LEED (lowenergy electron diffraction). A regular hexagon with zero reflection at the center in the LEED pattern is shown in Fig. 2 (inset). Since $\mathrm{SnAs}$ has the $\mathrm{NaCl}$ structure, the obtained pattern of reflections corresponds to the (111) plane. Clear reflection pattern also proves the single phase composition at the investigated surface.

Elemental composition of the sample was measured by EDS (Energy-Dispersive X-ray Spectroscopy) technique using SEM; the spectrum contains only lines related to the Sn- and As- atoms. Quantitative analysis shows Sn and As content of $48.9 \%$ and $51.1 \%$, respectively, that corresponds to the ratio of $0.978: 1.022$.

ARPES measurements were performed using a semispherical VG Scienta R4000 energy analyzer with energy resolution of $17 \mathrm{meV}$ and angular resolution of $0.5^{\circ}$. The ultraviolet helium lamp VUV 5k was used as a light source with photon energy $21.2 \mathrm{eV}$. The measurements were carried out at room temperature. Pressure in the 
chamber didn't exceed $1-2 \times 10^{-10}$ mbar. The samples were cleaved along the (111) plane (see the LEED pattern on Fig. 2, inset, as described above). Before measuring photoelectron spectra, the surface of the samples was cleaned by Ar-ion beam and subsequent heating at temperature of $150^{\circ} \mathrm{C}$ under ultrahigh vacuum.

Resistivity, magnetic and thermal properties of SnAs sample were measured using PPMS-9 (Quantum Design). Specific heat measurements of SnAs crystals were carried out by thermal relaxation technique with ${ }^{3} \mathrm{He}$ calorimeter, in the range of temperatures $0.4-6 \mathrm{~K}$ and magnetic fields 0 - 500 Oe. Low-frequency magnetic susceptibility measurements of SnAs crystals were taken in the range of temperatures $2-6 \mathrm{~K}$ and magnetic fields $0-200 \mathrm{Oe}$. It is worth of noting, that magnetic susceptibility at " $\mathrm{H}=0$ " is actually measured at a finite low value $(2-5 \mathrm{Oe})$ of the AC-magnetic field while the external DC-magnetic field is set to zero. Magnetization loop was measured using Vibrating Sample Magnetometer (VSM) at temperature of $2 \mathrm{~K}$. For thermal and magnetic measurements we used a sample with mass of $6.66 \mathrm{mg}$.

In order to implement the Andreev reflection (AR) spectroscopy, the SnS contacts were prepared in situ using the break-junction technique [20, 21]. In this technique, a flat thin crystal is mounted on a flexible substrate and then is finely bent until it cracks at the preliminary notched narrowing. As a result, a cleft at the designated location is formed in clean cryogenic environment. After cleaving, using a fine micrometric screw, we adjusted the cryogenic cleavage until a desired Andreevor Josephson-type $I-V$ characteristic was obtained. During the cryogenic experiment we tuned mechanically the cleavage, and probed, respectively, different microcontacts in order to select a high-transparency contact with the required type $I-V$ characteristic. In order to take four-contact transport measurements the sample was placed on top of the thin printed circuit board (PCB) made of FR-4 textolite; the PCB, in turn, was fixed on a flat Be-bronze springy holder, made of $0.1 \mathrm{~mm}$ thick foil. The sample was fixed to PCB with In-clamping contacts, reinforced with silver paste. The springy holder was finely bent by a tip of the micrometric screw pushing the holder from the side opposite to the sample. This design enabled us to cleave the sample and precisely vary the distance between the cleft banks during the cryogenic experiment.

It is a common knowledge, that the $d I / d V$ characteristics of high transparency superconductor - normal metal - superconductor $(\mathrm{SnS})$ microcontacts exhibit a so called subharmonic gap structure [22], comprising characteristic dips at bias voltages $V_{n}=2 \Delta / n e$ with $n$ being the subharmonic number. The subharmonic order $n$ equals to the number of Andreev reflections the carriers experience when moving in the normal constriction of the $\mathrm{SnS}$ junction. Therefore, the superconducting gap value $\Delta$ may be determined directly from the measured $V_{n}$ ver- sus $1 / n$ dependence.

The $d I / d V$ measurements were made in the temperature range from 1.5 to $4 \mathrm{~K}$. The measurements were taken by four probe technique. In order to bias the sample we used a voltage controlled current source fed with a sum of the $\mathrm{DC}$ bias and $\mathrm{AC}$ modulation voltages. The running current and voltage were measured independently using a precise digitizer; $d I$, and $d V$ were obtained using two lock-in amplifiers SR-830.

\section{RESULTS AND DISCUSSION}

\section{DFT/GGA calculations}

Theoretical band structure for SnAs was calculated within density functional theory (DFT) with the fullpotential linear augmented plane-wave framework as implemented in WIEN2k 23] together with the generalized gradient approximation (GGA) by Perdew, Burke and Ernzerhof 24] to the exchange-correlation functional. These calculations were converged self-consistently on a grid of $1000 k$-points in the irreducible Brillouin zone. Calculations were performed both, with and without spin-orbit coupling (SOC). The SOC was considered in a second-variational procedure [25]. Presented below DFT/GGA results for bulk SnAs agree well with those reported earlier in Refs. [15, 18]

The DFT calculations for bulk SnAs were based on the space symmetry group $F m \overline{3} m$ (No. 225) and lattice constant obtained in this work. Also, to consider real experimental geometry of the samples we have constructed (111) slab with topmost layer of Sn (vacuum gap between slabs is $25 \AA$ ). The (111) slab crystal structure is found to have monoclinic $C 2 / m$ (No. 12) space symmetry group. Calculations were performed for slabs with thickness of 5 and 2 unit cells (UC). No difference between the band structure was found for $5 \mathrm{UC}$ and $2 \mathrm{UC}$ slabs. Correspondingly, all discussed below results were obtained for $2 \mathrm{UC}$ (111) slab. Also, we have done structural relaxation for 2UC SnAs (111) slab, but have not seen any significant atomic position changes. The relaxation causes only minor modification of the electronic structure and is not exploited furthermore.

The DFT/GGA results for SnAs are presented on Fig. 3. Panel a) shows the total, and panels b) and c) - partial density of states for bulk SnAs. A mixture of various electronic states (Sn-5s, Sn-5p and As-4p) is observed near Fermi level with a slight predominance of As-4p states. As a result, the Fermi level lies in the recess of density of states.

SOC impact on the DOS is most pronounced for As-4p states in the interval between $-0.5 \mathrm{eV}$ and $-2 \mathrm{eV}$ as follows from the dotted line on panels c) and g). Also, SOC slightly affects the Sn-5p states. The same effect is visible for dispersions along $\mathrm{X}-\mathrm{\Gamma}-\mathrm{L}$ directions on panels $\mathrm{d}$ ) 

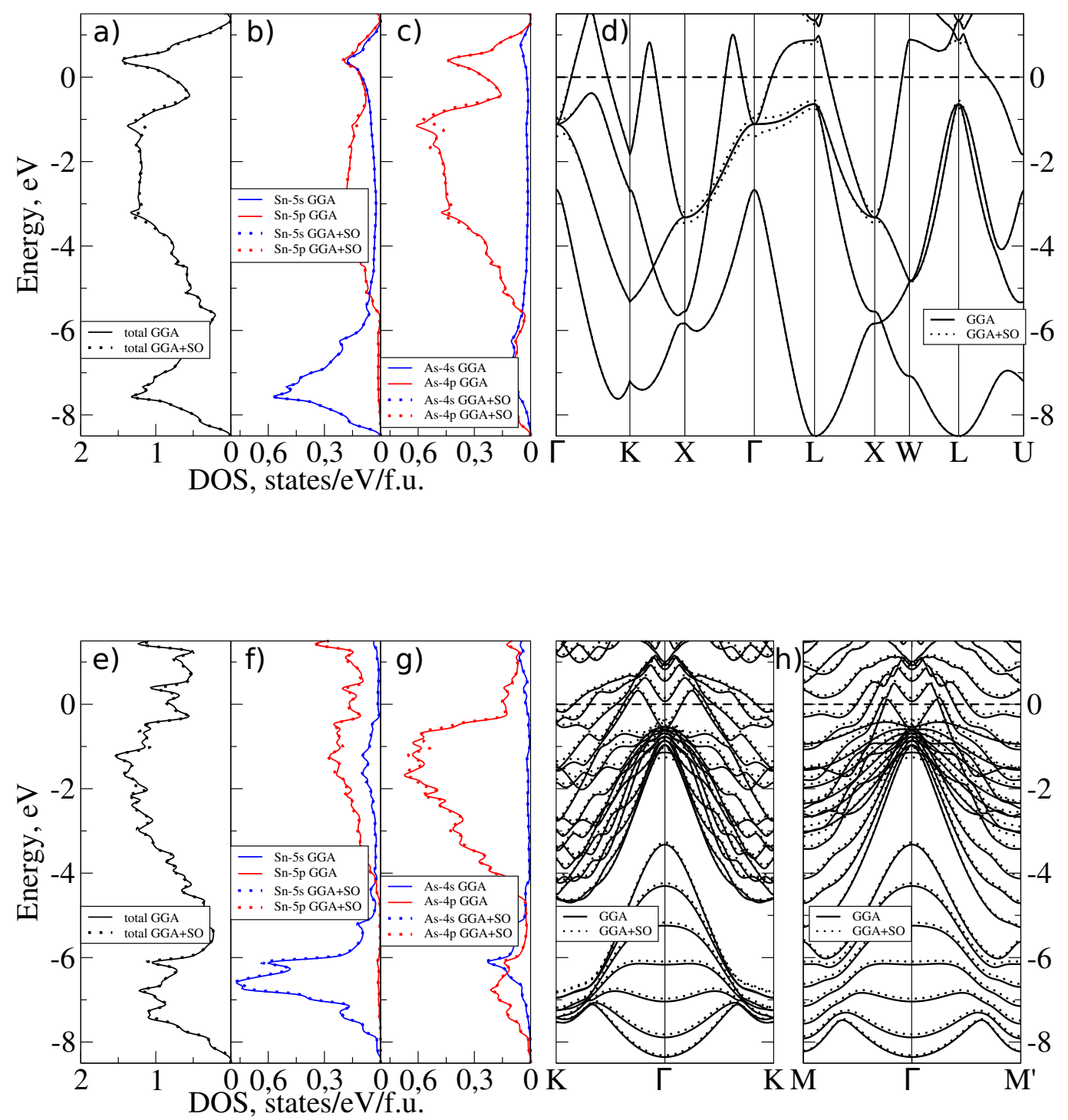

Figure 3. DFT/GGA calculated total and partial densities of states [panels (a),b), c), e), f), g)] and band dipersions for the bulk [panel d)] and for the slab [panel h)] crystal structures. Dotted lines denote the DFT/GGA calculations with SOC and solid lines - without SOC. Zero energy corresponds to the Fermi level.

and h) of Fig. 3. Other bands are practically not affected by SOC. The strongest manifestation of SOC can be observed near $\Gamma$-point at about $-1 \mathrm{eV}$ and provides lifting of the bands degeneracy with the splitting by a few tens of $\mathrm{eV}$, in a qualitative agreement with earlier calculations [18, 19]. Concerning possible link between SOC and superconductivity in SnAs, one can see that SOC doesn't manifest considerably in the vicinity of the Fermi level. Therefore, one should not expect strong influence of SOC on superconductivity.

Several observations based on the DFT/GGA results should be mentioned regarding the superconductivity character in SnAs. Firstly, bulk DFT/GGA band structure of SnAs (Fig. 3 (d)) has many bands crossing the Fermi level. It supposes SnAs to be a multiband superconductor and, in the normal state, a good threedimensional metal. The shape of the calculated Fermi surface presented on Fig. 4 confirms the last two statements. Thirdly, the total density of states at the Fermi level is rather small - 0.81 states/eV/f.u. (f.u. - formula unit) which might lead to experimentally observed $\operatorname{low} T_{c}$.

From Fig. 3 (d) and Fig. 4one can see that electronic spectra have a complicated anisotropic character. There 


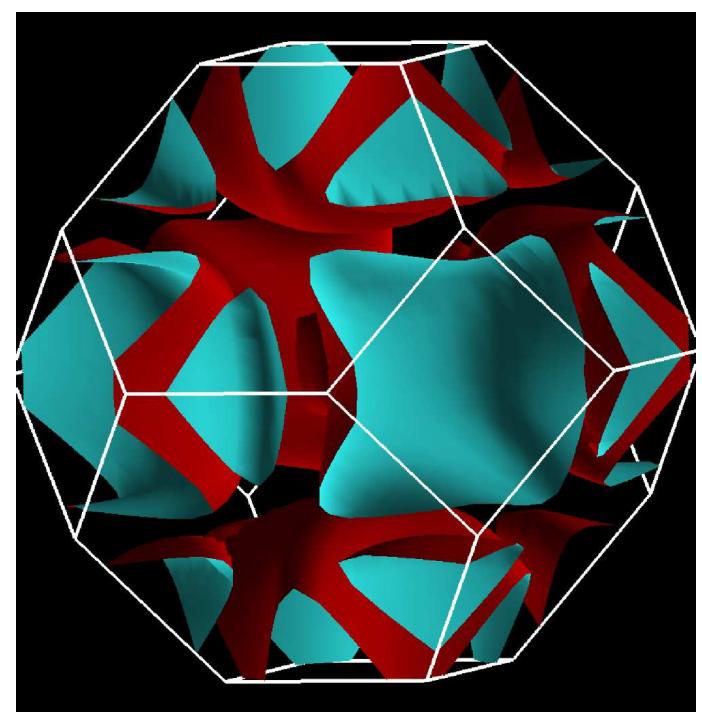

Figure 4. SnAs DFT/GGA calculated Fermi surface.

are 10 different Fermi level crossings, each has its particular Fermi momentum $k_{F}$ and effective electron mass $m^{*}$. Since SnAs with a large Fermi surface is a pretty good metal, for furher analysis we employ the Fermi liquid type description and deduce from DFT/GGA calculations several parameters important for the superconducting state. Firstly, we estimated the effective mass enhancement through the specific heat linear coefficient $\gamma_{\text {calc }}=\frac{\pi^{2}}{3} N_{A} k_{B}^{2} N\left(E_{F}\right)=1.89 \mathrm{~mJ} /\left(\operatorname{mol} \times \mathrm{K}^{2}\right)$, where $N_{A}$ is the Avogadro constant, $k_{B}$ - Boltzmann constant and $N\left(E_{F}\right)$ - the DFT/GGA value of total DOS at the Fermi level. Then $\frac{\gamma_{n}}{\gamma_{\text {calc }}}=\frac{m^{*}}{m_{e}}$ with $\gamma_{n}=2.67 \mathrm{~mJ} /\left(\mathrm{mol} \times \mathrm{K}^{2}\right)-$ electronic specific heat in the normal state (see below) gives $\frac{m^{*}}{m_{e}}=1.41$. This ratio agrees well with Ref. [18] despite both calculated $\gamma_{\text {calc }}$ and experimental $\gamma_{n}$ exceed by a factor of $\sim 1.2$ the respective values used in Ref. [18].

Specific heat, effective mass and Fermi momentum may be characterized, to the first approximation, with their single values averaged over the Fermi surface despite complicated multi band character of electron dispersions. Finding such averaged parameters for arbitrary non-elliptic Fermi surface (see Fig. (4) is rather difficult. One can try to obtain $k_{F}$ value again using $N\left(E_{F}\right)$ - the calculated value of the total DOS at the Fermi level. Then $k_{F}=N\left(E_{F}\right) \frac{4 \pi^{2} \hbar^{2}}{V \frac{m^{*}}{m_{e}} m_{e}}=0.6 \AA^{-1}$, where $V$ is elementary cubic cell volume. Also it may be found as $k_{F}=\left(3 \pi^{2} n\right)^{1 / 3}$, where $n=N / V$ with $N$ - number of valence electrons in the unit cell. From partial DOS one can see that there are 2.1 of As- $4 \mathrm{p}$ electrons, 0.1 Sn-5s electrons and 0.72 Sn-5p electrons; hence, in total $N$ per formula unit is 2.92 . There are 4 formula units per cubic cell with $V=187.5 \AA^{3}$. It gives $k_{F}=1.23 \AA^{-1}$. Such discrepancy between $k_{F}$ values comes from multi- band anisotropic nature of SnAs spectra while all definitions above are for the isotropic single band Fermi liquid. There is also theoretical conjecture for concentration in Ref. 13] $\mathrm{n}=2.14 \times 10^{22} \mathrm{~cm}^{-3}$, which gives $k_{F}=0.86 \AA^{-1}$.

Previously these values were estimated in the Ref. 14] to be $m^{*}=1.20 m_{e}$ and $k_{F}=1.24 \AA^{-1}$ respectively within a straightforward single-sheet spherical Fermi surface approach. Here, from the DFT/GGA calculations we see that the Fermi surface has many sheets and is rather anisotropic. Although the values of $m^{*}$ and $k_{F}$ obtained here on the basis of DFT/GGA calculations are surprisingly quite similar to those of the Ref. [14].

Lower panels e) to h) in Fig. 3 present DFT/GGA results for (111) slab. One can see that the total and partial DOS are practically the same as those for bulk SnAs. The value of total DOS at the Fermi level also is nearly the same as the bulk one. Manifestations of SOC are weak, however the band dispersions are rather different. First of all, the difference comes from different paths in $k$-space for bulk and (111) slab as shown on Fig. [5(a). Secondly, there are much more bands for the slab since $k_{z}$ translation invariance is broken for the surface. As a result, all atoms even of the same sort become inequivalent and produce their own set of bands. Nevertheless, in general SnAs bulk band shape to some extent resembles that for (111) slab crystal structure.

\section{ARPES vs. DFT/GGA}

Earlier ARPES results obtained by our group were reported in Ref. [19]. We noted there the observed band bundle spliting at the $\Gamma$-point not captured by the existed DFT calculations [15, 18]. We suggested this disagreement to be caused either by SOC, or by a band structure reconstruction on the crystal surface 19. We now compare ARPES data and DFT/GGA calculated band dispersions to clarify these issues and conclude that the reason of the band splitting is indeed the reconstruction of the band structure at the crystal surface [19].

Bulk calculations for $k_{z}$ other than 0 (the $k_{z}$ direction coincides with the [111] direction) show, that by varying $k_{z}$ it is not possible to improve agreement between the calculated and experimental spectra, similar to a number of studies [26, 27]. In this regard, the work presents the calculation results for the case $k_{z}=0$.

In Fig. 5 panels (b) and (e) show ARPES data and DFT/GGA bands for bulk SnAs without and with SOC, respectively. The SnAs bulk DFT/GGA bands resemble ARPES data very remotely. There is a bundle of bands around $1.1 \mathrm{eV}$ (predominantly of As-4p character) in calculated and ARPES data. However, DFT/GGA shows only three bands in the bundle plus one band by $1.5 \mathrm{eV}$ lower, whereas ARPES clearly demonstrate four bands in the vicinity of the $\Gamma$-point. Also energy positions of those bands are quite different in ARPES and 

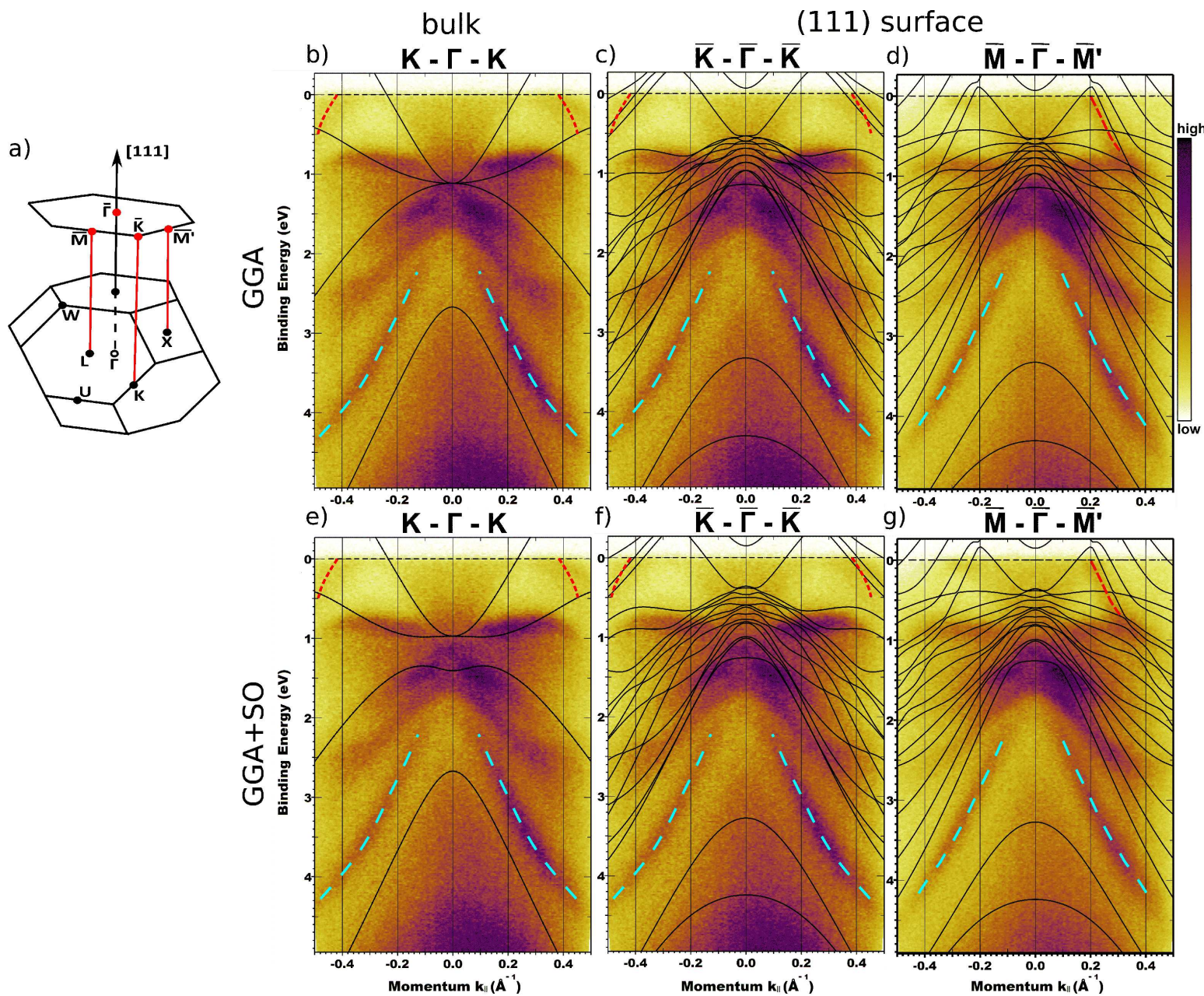

g) $\stackrel{-0.4}{-0.2} \overline{\mathrm{M}}=\frac{0.0}{\bar{\Gamma}}-\frac{0.2}{\mathrm{M}^{\prime}}$

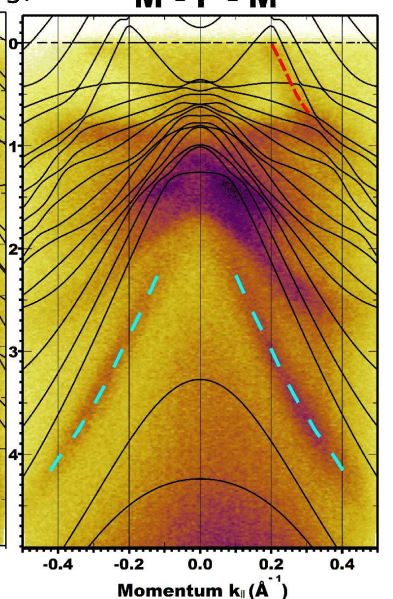

Figure 5. a) The first Brillouin zone for the NaCl-type structure with the projection to the (111) plane. b), e) calculated bulk band structure (solid lines) and ARPES spectra (contour plots) for SnAs along $\mathrm{K}-\Gamma-\mathrm{K}$ direction. c), d), f), g) ARPES spectra with DFT/GGA calculated band structure of (111) slab along high symmetry directions $\overline{\mathrm{K}}-\bar{\Gamma}-\overline{\mathrm{K}}$ and $\overline{\mathrm{M}}-\bar{\Gamma}-\overline{\mathrm{M}}^{\prime}$ from panel (a). The lower row corresponds to DFT/GGA calculations with SOC. Dashed lines near Fermi level show maxima of the ARPES data maps. Zero energy corresponds to the Fermi level.

DFT/GGA. Somehow ARPES does not resolve bands crossing the Fermi level, the most intensive signal is in the vicinity of the $\Gamma$-point (the momenta interval from -0.2 to $0.2 \AA^{-1}$ of the ARPES spectra, Fig. (5).

Inclusion of SOC splitting slightly improves the situation. The wings around 0.9 and $1.3 \mathrm{eV}$ agrees a bit better with experiment but ARPES does not show clear separation of those bands. One should note also that bulk DFT/GGA bands along $\mathrm{K}-\Gamma-\mathrm{K}$ direction do not reproduce ARPES bands near Fermi level shown by red dashed curves on ARPES spectra.

In case of (111) slab calculations, as panels (c,d,f,g) of Fig. [5 show, the calculations demonstrate the emergence of electronic dispersion which much better reproduce ARPES bands near the Fermi level shown with red dashed lines for both $\overline{\mathrm{K}}-\bar{\Gamma}-\overline{\mathrm{K}}$ and $\overline{\mathrm{M}}-\bar{\Gamma}-\overline{\mathrm{M}}^{\prime}$ directions. We conclude, the Fermi level crossing positions are well captured by DFT/GGA calculations in the vicinity of the Fermi level. Wings at about $0.9 \mathrm{eV}$ are well reproduced here too. Dark ARPES data region at $\Gamma$-point about $1.1 \mathrm{eV}$ below $E_{F}$ is qualitatively represented by bunch of DFT/GGA "spaghetti". Strictly speaking not all of these "spaghetti" may be seen by ARPES since at a given incident beam energy the penetration depth is about $7 \AA$. It corresponds to one, maximum two top most surface layers. The layers below should not provide significant ARPES signal. SOC has almost no effect on the shape of the DFT/GGA bands and does not bring about agreement with ARPES data. Based on the above comparison we conclude, that the ARPES spectra in general in the vicinity of $E_{F}$ are in good agreement with the DFT/GGA calculated bands for (111) slab of SnAs. However, the only one band with maximum at about $3 \mathrm{eV}$ (highlighted with light blue dashed curves in Fig.(5) isn't reproduced by any of the performed calculations. We suppose, that this feature might be a main 
theme of further studies.

Resistivity, specific heat and magnetic susceptibility

For more detailed information about superconducting properties of SnAs we performed resistivity, specific heat and magnetic susceptibility measurements. Temperature dependence of resistivity is shown in Fig. 6 and demonstrates the metallic-type conduction of the sample, which is consistent with the band structure calculations and ARPES data (Fig. (5)). At a temperature of about $3.6 \mathrm{~K}$ (for sample No.1), the resistivity shows a transition to the superconducting state (Fig. 6, inset). This agrees with the earlier data [14].

Temperature dependence of the magnetic AC susceptibility $\chi$ of SnAs crystal is presented in Fig. 7 (for sample No.2). The susceptibility for this sample demonstrates superconducting (SC) transition near $3.8 \mathrm{~K}$ in zero field, slightly higher than the SC transition in transport (for sample No.1). As the applied DC magnetic field increases, the superconducting transition temperature gradually decreases and an additional positive peak appears in the $\chi(T)$ dependence near $T_{c}$. This peak corresponds to the so called differential paramagnetic effect (DPE) in superconductors 28 30]. DPE (positive $\left.\chi_{a c}\right)$ corresponds to the existence of a region with strong growth in $M(H)$ dependence (and, as a consequence, positive derivative $\partial \mathrm{M} / \partial \mathrm{H})$. This effect is observed for mixed state of type-I superconductors or in type-II superconductors with close $H_{c 1}$ and $H_{c 2}$ values.

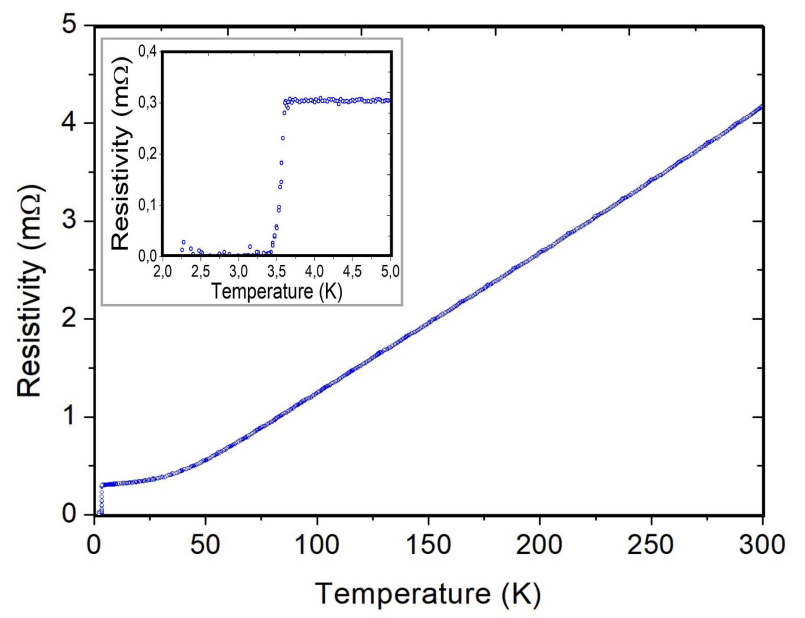

Figure 6. Temperature dependence of resistivity for SnAs (sample No.1). The inset shows the zoomed-in region of superconducting transition.

Figure 8 shows isothermal magnetization loop $M(H)$ at $T=2 \mathrm{~K}$. The shape of the curve is nearly typical for type-I superconductor, however it reveals a small hysteresis. Such hysteresis is observed in other compounds

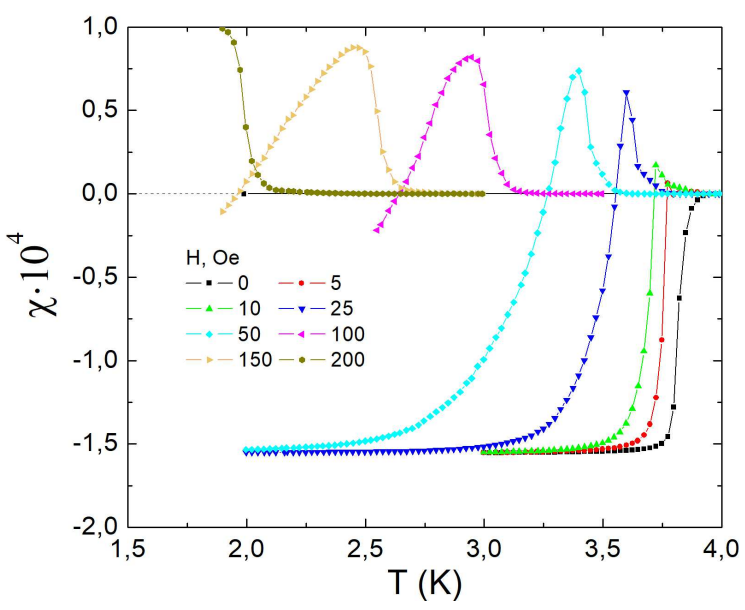

Figure 7. AC-Magnetic susceptibility of the SnAs (sample No. 2).

considered to be type-I superconductors and, generally, is associated with sample shape effects, domain or grain walls etc 29,3134 .

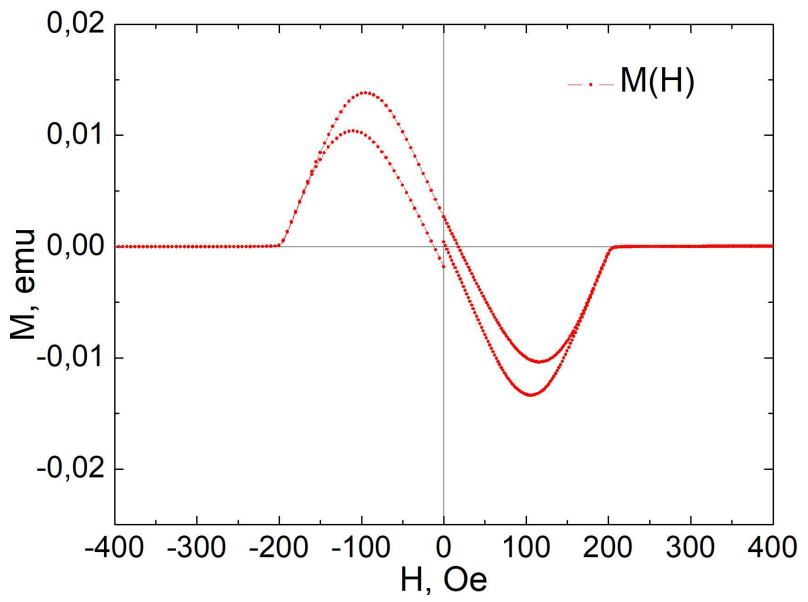

Figure 8. Isothermal magnetization loop $M(H)$ at $2 \mathrm{~K}$ (sample No. 2).

Temperature dependences of the specific heat in various magnetic fields are shown on Fig. 9 The sharp jump in specific heat at $H=0$ for temperature near $4 \mathrm{~K}$ confirms the bulk superconducting transition. From the local entropy conservation we find the critical temperature $T_{c}=3.80 \pm 0.05 \mathrm{~K}$, consistent with susceptibility measurements. The total width of the $\mathrm{SC}$ transition $\Delta T_{c}=0.15 \mathrm{~K}$ is another evidence of the high quality of the studied crystal. In general, our data for zero field specific heat is reasonably consistent with 14.

Specific heat tends to zero at $T \longrightarrow 0$ in zero field and does not demonstrate any anomalies in the low-temperature region. This tendency is an additional evidence of bulk nature of superconductivity in 
the studied samples. The polynomial extrapolation to zero temperature $C(T) / T=\beta T^{2}+\gamma_{r} \quad(\beta=0,317 \pm 0,019$ $\left.\mathrm{mJ} /\left(\mathrm{mol} \times \mathrm{K}^{4}\right)\right)$ gives residual electronic specific heat $\gamma_{r}=0.015 \mathrm{~mJ} /\left(\mathrm{mol} \times \mathrm{K}^{2}\right)$.

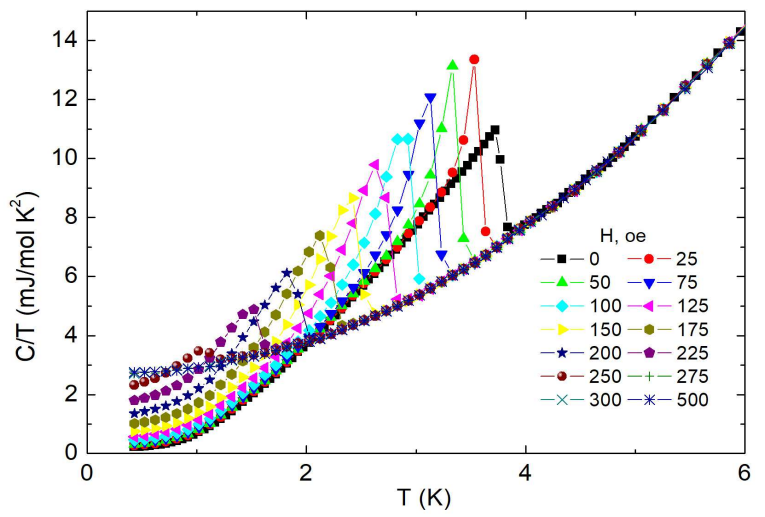

Figure 9. Temperature dependences $C(T) / T$ for the SnAs crystal (sample No. 2) in magnetic fields $0-500$ Oe.

Magnetic field suppresses the superconducting transition and shifts it gradually to lower temperatures. At fields above $300 \mathrm{Oe}$, the superconductivity is fully suppressed, as follows from the equality of the specific heat at $H=300$ Oe and higher fields, for example, 500 Oe. Thus, the electronic component of the specific heat can be defined as $C_{e}=C(H)-C\left(H_{0}\right)+\gamma_{n} T$ for $H<H_{0}$, (where $H_{0}=300$ Oe exceeds the critical magnetic field), taking into account the entropy conservation. The electronic specific heat in the normal state $\gamma_{n}$ at temperatures above $T_{c}$ was found to be $\gamma_{n}=2.67 \mathrm{~mJ} /\left(\operatorname{mol} \times \mathrm{K}^{2}\right)$.

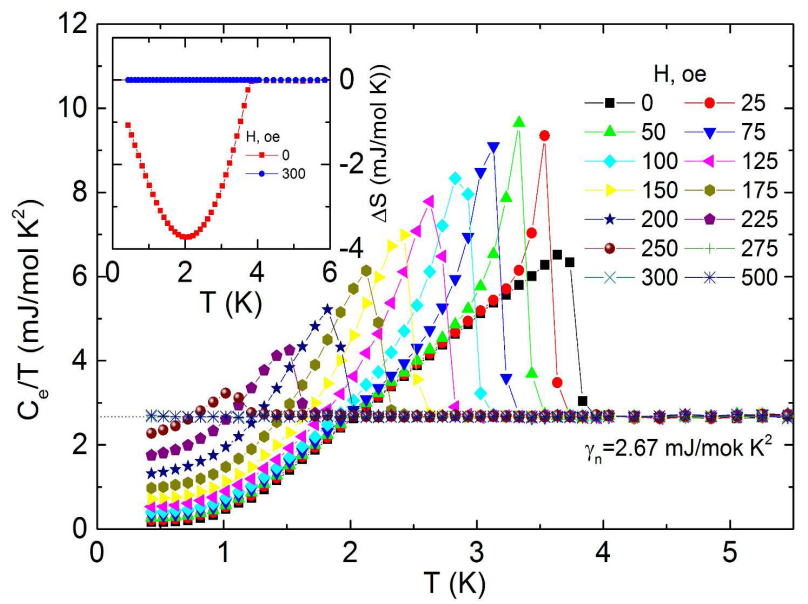

Figure 10. Electronic specific heat $C_{e}(T) / T$ in fields $0-$ 500 Oe (sample No. 2). Inset: the difference between the entropy in superconducting state at zero field and normal state (at $H=300$ Oe) $\Delta S=S_{s}-S_{n}$.

The residual term $\gamma_{r}$ is much less than the electronic specific heat in the normal state $\gamma_{n}$. The ratio $\gamma_{r} / \gamma_{n} \approx$

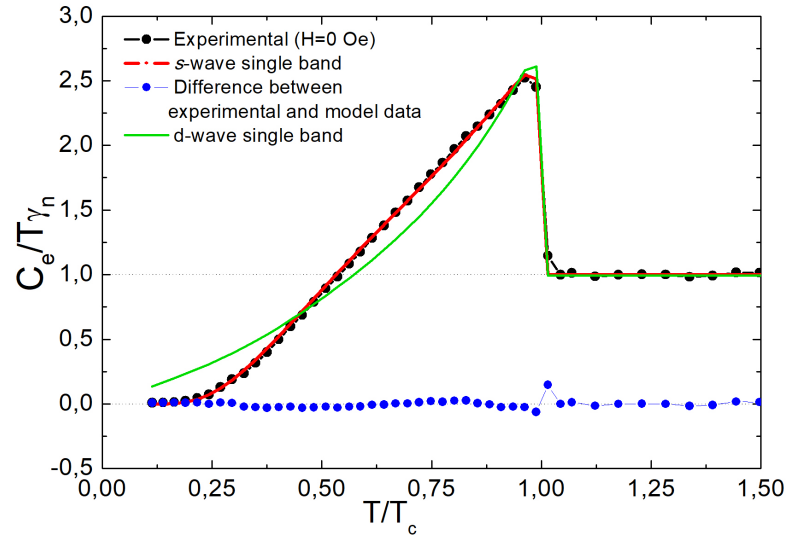

Figure 11. Normalized electronic specific heat of the superconducting condensate $\frac{C_{e}}{T \gamma_{n}}$ and its best fit with the s- and d- wave $\alpha$-model (sample No. 2).

$0.5 \%$ quantifies the relative amount of unpaired carriers; it confirms the bulk character of the superconducting state, and evidences for a high crystal purity.

Temperature dependence of the electronic contribution to specific heat $C_{e}(T) / T$ in various fields is shown in Fig. 10 .

Inset of Fig. 10 shows difference between entropy in superconducting and normal states. We now estimate critical magnetic field value from the obtained data. It could be done from equation $w=\frac{|\Delta F| \cdot N}{V}=\frac{H_{c}^{2}}{8 \pi}$, where $w$ is the density of energy, $\Delta \mathrm{F}$ is the difference between free energies in superconducting and normal states, $\mathrm{N}$ is the molar quantity, and $\mathrm{V}$ is the sample volume. Numerical integration give us $\Delta F=\int_{0}^{T_{c}} \Delta S d T=-9.00 \mathrm{~mJ} / \mathrm{mol}$, where $\Delta S$ is the difference between the entropy in superconducting state at zero field and in normal state. Taking into account $\mathrm{N}=\mathrm{m} / \mu=3.44 \cdot 10^{-5} \mathrm{~mol}$ (here $\mu$ is the formula mass of SnAs, $\mathrm{m}=6.66 \mathrm{~g}$ is the sample mass) $\mathrm{V}$ $=\mathrm{m} / \rho=0.971 \cdot 10^{-3} \mathrm{~cm}^{3}\left(\rho=6.86 \mathrm{~g} / \mathrm{cm}^{3}\right.$ is the mass density) we have for critical magnetic field $\mathrm{H}_{c}=283$ Oe.

To analyze thermal properties of superconducting condensate, it is convenient to consider the normalized electronic specific heat $C_{e n}=\frac{C_{e}}{T \gamma_{n}}$. For the superconducting condensate it may be calculated within the framework of the BCS theory using the so-called alpha model 35]. The $\alpha$-model may be generalized in case of possible in-plane anisotropy (d-wave $\alpha$-model) as presented in [36], with two adjustable parameters $\alpha=\frac{2 \Delta(0)}{k_{B} T_{c}},(\Delta(0)$ is the superconducting gap at zero temperature), and $m(\varphi)=1+\mu \cos (2 \varphi)$ - angular dependence of the gap.

Normalized electronic specific heat of the superconducting condensate $\frac{C_{e}}{T \gamma_{n}}$ versus $\left(T / T_{c}\right)$ and its best fit with the s- and d- wave $\alpha$-model [35] are presented 
in Fig. 11. The simplest s-wave model (isotropic gap, $m(\varphi)=1$ ) has only one free parameter $\alpha$. The best description of the experimental data was obtained with $\alpha$ $=3.73$ which is very close to the characteristic BCS ratio $(\alpha=3.52)$ and corresponds to the superconducting gap of $\Delta=0.6 \mathrm{meV}$ (see Fig. 111). Clearly, the model with the d-wave gap symmetry $(m(\varphi)=\cos 2 \varphi)$ is at odd with the experimental data of Fig. 11] Fitting with the extended s-wave gap symmetry $(m(\varphi)=1+\mu \cos (2 \varphi))$ leads to $\mu \rightarrow 0$, and therefore reduces to the simple s-wave model with an isotropic gap. The same result is obtained for the two-band $\alpha$-model which corresponds to the two-band superconductivity 35]: $C(T)=\varphi_{1} C_{1}(T)+\varphi_{2} C_{2}(T)$. In this case from the fitting procedure, the contribution of one of the gaps $\varphi_{1}$ becomes equal to zero, whereas angular modulation for the second one vanishes $(\mu \longrightarrow 1)$.

Evidently, the best description of the experimental data is obtained using the single-band BCS model with an isotropic gap and $\alpha=\frac{2 \Delta(0)}{k_{B} T_{c}}=3.73$. Despite SnAs as shown above has essentially multi band electronic system application of single band BCS model can be justified in case matrix of coupling constant consists of approximately equal values for all Fermi surface sheets [37].

Figures 9 and 10 show temperature dependence of specific heat in magnetic field. Suppression of the specific heat jump at $\mathrm{H}=500 \mathrm{Oe}$ was also reported in Ref. [14], however, the temperature dependence of specific heat in lower magnetic fields wasn't explored there. For nonzero fields, beside the conventional jump at $T_{c}(H)$, the electronic specific heat demonstrates an additional sharp peak near the SC transition (see Figs. 9] and 10). Commonly, such feature is considered as a transformation of the 2 nd- to the 1st- order (in the presence of a magnetic field) phase transition in type-I superconductors, such as, e.g., thallium and aluminum [38, 39 as well as for typeI compound superconductors [29, 31, 33]. The increase in the specific heat near the superconducting transition signifies, that an additional energy is required to realize the superconducting transition in magnetic field.

Similar behavior may be also observed for other firstorder phase transitions: e.g., for melting transition, where the specific heat of a crystal changes to the specific heat of a liquid right at the melting point, however, the temperature does not change during melting despite the heat enters the system; as a result, the specific heat exhibits a sharp peak. In our case, similarly, the transition from superconducting to normal state occurs with the absorption of latent heat. The finite width of the peak is due to the intermediate state (shape effect). Thus, the specific heat in magnetic fields demonstrates features intrinsic to the first-order phase transition and indicates SnAs to be the type-I superconductor.

Figure 12 shows magnetic field dependence of the superconducting transition temperature, determined from the AC susceptibility and specific heat data. The dashed

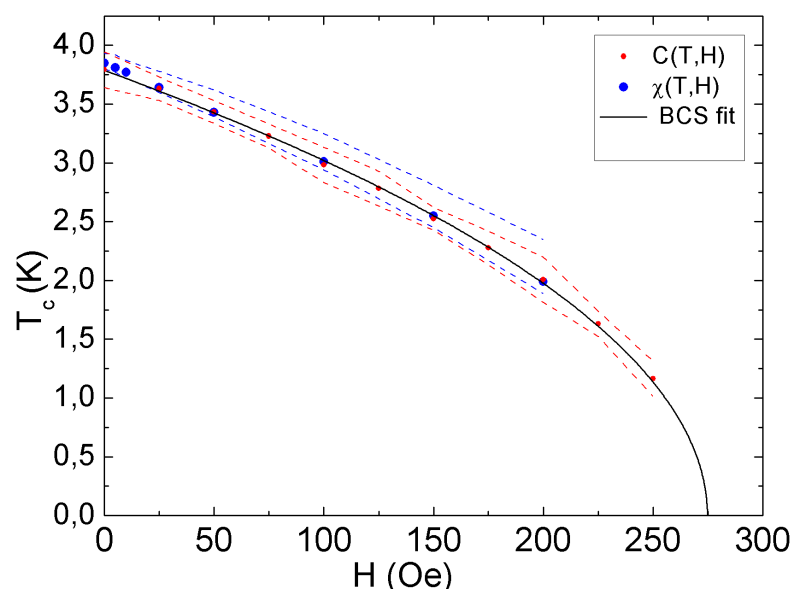

Figure 12. Critical temperature $T_{c}$ vs magnetic field, determined from the AC susceptibility and specific heat; solid line represents the BCS parabolic fit $H_{c}(T)=H_{c}(0)\left(1-\left(T / T_{c}\right)^{2}\right)$ (sample No. 2).

lines depict the corridor between the beginning and ending of the transition (red lines and dots correspond to the specific heat data and blue ones - to the AC susceptibility). The points correspond to the mean values. The mean $T_{c}(H)$ data obtained from the specific heat coincides with that obtained from the susceptibility measurements and slightly exceeds the resistivity data. This dependence is well described by the BCS dependence $H_{c}(T)=H_{c}(0)\left(1-\left(T / T_{c}\right)^{2}\right)$ that extrapolates to $H_{c}(0)=275$ Oe; the latter value is reasonably consistent with $H_{c}=283$ Oe, estimated from the difference in entropy between superconducting and normal states.

The data obtained enables one to estimate superconducting parameters of the SnAs compound. Electronic part of specific heat in normal state together with $\mathrm{k}_{F}$ and $m^{*}$ found from DFT/GGA results described above give possibility to estimate London penetration depth $\lambda_{L}$, coherence length $\xi$, and Ginzburg-Landau parameter $\kappa$. For calculations we take DFT/GGA based Fermi momenta $\mathrm{k}_{F}$ from 0.6 to $1.23 \AA^{-1}$, while $m^{*}=1.41 \mathrm{~m}_{e}$ is rather safely obtained from DFT/GGA and specific heat.

Then London penetration depth may be estimated using formula $\lambda_{L}(0)=\left(3 \pi^{2} m^{*} /\left[\mu_{0} k_{F}^{3} e^{2}\right]\right)^{1 / 2}$. It gives $\lambda_{L}(0)$ in the range from 25.7 to $75.4 \mathrm{~nm}$. Corespondingly, coherence length $\xi(0)=0.18 \hbar^{2} k_{F} /\left(k_{B} T_{c} m^{*}\right)$ equals to $171.0-350.5 \mathrm{~nm}$ [40] and Ginzburg-Landau parameter $\kappa=\lambda_{L}(0) / \xi(0)=0.07-0.4<1 / \sqrt{2}$; the latter confirms our conclusion on the type I superconductivity for SnAs. For a particular choice $k_{F}=1.23 \AA^{-1}$, our results are close to those of Ref. [14]. 


\section{Andreev reflections spectroscopy}

In order to have a deeper insight into the superconducting properties of SnAs and, particularly, temperature dependence of the superconducting energy gap $\Delta$, we measured Andreev reflection spectra. The superconductor - normal metal - superconductor ( $\mathrm{SnS}$ ) Andreev reflection spectroscopy is a powerful tool to determine the energy gap value and its temperature evolution. Unlike several other intimate techniques, such as scanning tunneling spectroscopy (STS), and point-contact Andreev reflections (PCAR), this technique does not require any data fitting [41, 42] or a model for data interpreting. Recently, Andreev reflections spectroscopy on symmetric SnS contacts was successfully used to quantify the gap structure for several multi-gap superconducting arsenides 43 45].
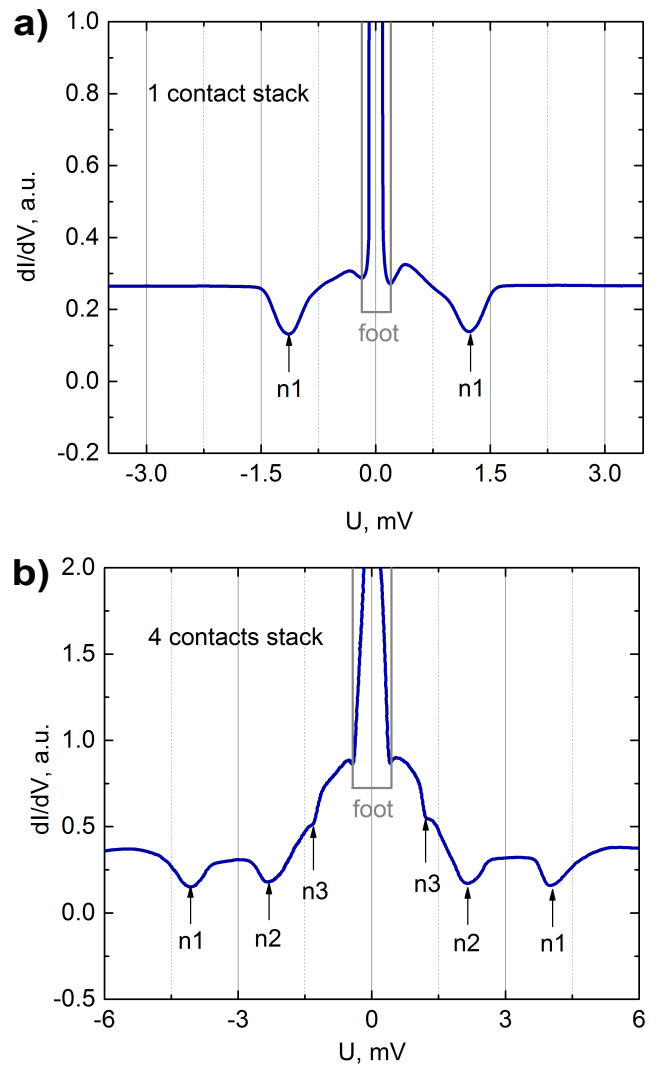

Figure 13. Dynamic conductances, measured at $\mathrm{T}=1.5 \mathrm{~K}$ for a single $\mathrm{SnS}$ contact (a) and for a 4-contact stack (b) (sample No. 1).

Figure 13(a) shows the dynamic conductance $d I / d V$ of a single Andreev-type contact, measured at $T=1.5 \mathrm{~K}$. The two symmetric dips in $d I / d V$ marked with label $n_{1}$ in Fig. 133(a) correspond to bias voltages $V_{1}= \pm 2 \Delta / n_{1} e$. No other features are seen at higher bias, and we con- clude that the dips correspond to the major $n_{1}=1$ resonance. From this representative data we obtain an estimate $\Delta \approx 0.55 \mathrm{meV}$. Andreev reflection dips of higher order may not be seen on this contact due to the following reasons. According to Ref. [42], the number of possible Andreev reflections is limited by the ratio $2 a / l$, where $a$ - is characteristic dimension of the constriction, $l$ - is the mean free path. Another possible reason is that the second and higher-order reflection dips may be masked by the sharply growing "foot" (excess conductance at low biases, which is intrinsic to Andreev contacts).

By mechanical tuning the sample bending and thus, the break junctions area one can realize Andreev contacts with different $2 a / l$ ratio and also stacks of sequentially connected S-n-S-n...-S contacts [20]. For the chain of $m$ contacts, evidently, the resonant bias voltage $V_{n}=$ $m \times(2 \Delta / n e)$ is increased by a factor of $m$. Figure 13(b) shows dynamic conductance for such stack of several contacts. One can see 3 features, corresponding to 3 resonances. Having the preceding single-contact spectrum, as a reference, we immediately disentangle the spectrum Fig. 13(b) and find that it is produced by a chain of 3 sequential contacts. Indeed, the observed dips at 4.1, 2,15 and $1.35 \mathrm{meV}$ may be fitted with $n_{1}=1, n_{2}=2, n_{3}=3$ and the consistency with the single contact spectrum is obtained for $m=3$. Finally, based on the above data we refine the gap value, $\Delta=0.53 \pm 0.04 \mathrm{meV}$ and the extrapolated value $\Delta(T \rightarrow 0)=0.54 \mathrm{meV}$. We stress, that for all studied contacts we didn't observe signatures of the second gap.

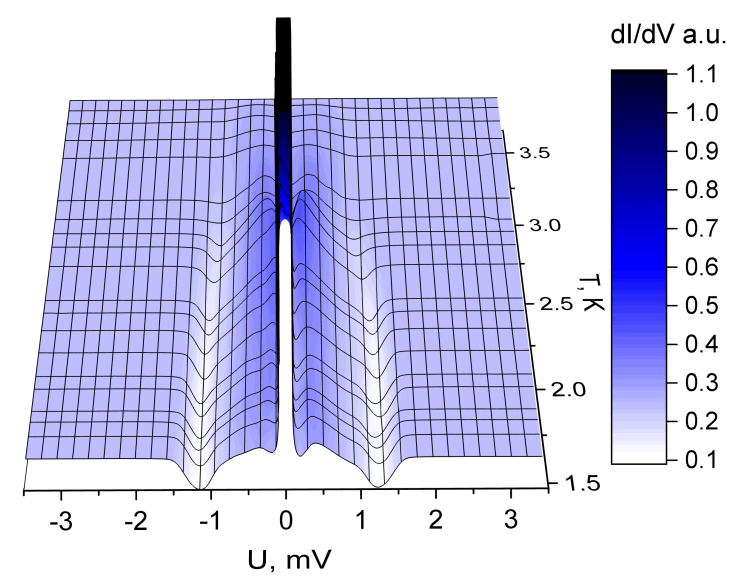

Figure 14. Temperature evolution of dynamic conductance for 1 contact stack a single Andreev contact (the peak at zero bias is cut off).

In order to find $\Delta(T)$ temperature dependence, we measured the dynamic conductance of a single contact in the range $1.5-4 \mathrm{~K}$. Figure 14 shows that with temperature rising, the dips in $d I / d V$ shrink towards zero bias and the dynamic conductance finally linearizes at 
temperature $\approx 3.6 \mathrm{~K}$, which is taken as the local critical temperature $T_{c}^{\text {local }}$.

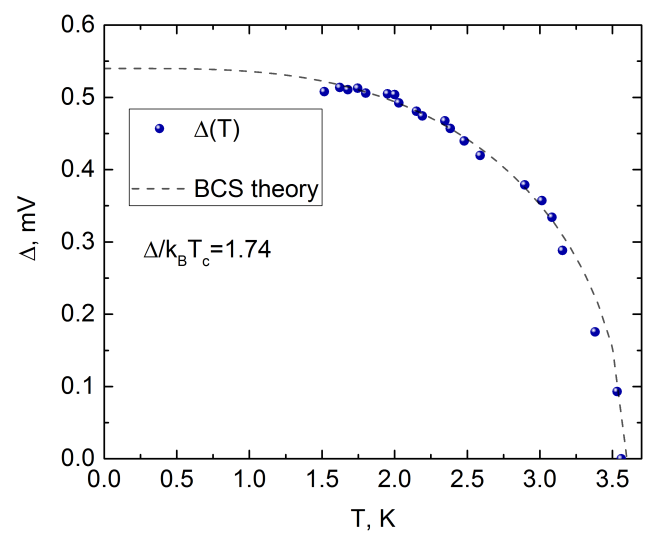

Figure 15. Energy gap temperature dependence deduced from the measured Andreev spectra. $\Delta=0.54 \pm 0.04 \mathrm{meV}$ is found from extrapolation of $\Delta(T)$ to $T=0$, obtained with the BCS fitting dependence.

Evolution of the gap value with temperature $\Delta(T)$ is plotted in Fig. 15. One can see that temperature dependence of the energy gap is well approximated by the single-gap BCS theory, and the ratio $2 \Delta(0) / k_{B} T_{c}=3.48$ signals the weak-coupling case.

\section{CONCLUSIONS}

\begin{tabular}{|c|c|c|}
\hline Parameters & $\overline{\text { Values }}$ & Values from Ref.[14] \\
\hline $\mathrm{a}, \ddot{A}$ & 5.723 & $5.72513(4)$ \\
\hline$\rho, \mathrm{g} / \mathrm{cm}^{3}$ & 6.86 & - \\
\hline$T_{c}, \mathrm{~K}$ & $\begin{array}{l}3.6 \text { (sample No. } 1) \\
3.8(\text { sample No. } 2)\end{array}$ & 3.58 \\
\hline $\mathrm{H}_{c}, \mathrm{Oe}$ & $\begin{array}{c}283 \text { (entropy) } \\
275 \text { (extrapolation) }\end{array}$ & 178 (extrapolation) \\
\hline$\Delta(0), \mathrm{meV}$ & $\begin{array}{c}0.6 \text { (specific heat) } \\
0.53 \pm 0.04(\mathrm{AR})\end{array}$ & - \\
\hline $2 \Delta(0) / T_{c}$ & $3.48-3.73$ & - \\
\hline$\gamma_{r}, \mathrm{~mJ} /\left(\mathrm{mol} \times \mathrm{K}^{2}\right)$ & 0.015 & - \\
\hline$\gamma_{n}, \mathrm{~mJ} /\left(\mathrm{mol} \times \mathrm{K}^{2}\right)$ & 2.67 & 2.18 \\
\hline$\beta, \mathrm{mJ} /\left(\mathrm{mol} \times \mathrm{K}^{4}\right)$ & 0.317 & 0.30 \\
\hline
\end{tabular}

Table I. Structural and thermodynamic data for SnAs in the normal and superconducting states. Parameters $\mathrm{H}_{c}, \gamma_{r}, \gamma_{n}$, $\beta$, and $\Delta(0)$ (specific heat) were obtained for sample No. 2, $\Delta(0)(\mathrm{AR})$ - for sample No.1. Interval of $2 \Delta(0) / T_{c}$ include parameters for both samples.

In summary, we performed comprehensive study of the band structure and superconducting properties of the SnAs binary compound. DFT/GGA band structure calculations were carried out for both bulk and (111) slab crystal structures with and without SOC. Our calculated spectra for bulk SnAs are in a good agreement with previous theoretical results. Experimental investigation of SnAs band structure was done by ARPES technique. Comparison of the ARPES results and band structure calculation shows that the calculated (111) slab band structure much better agrees with ARPES data than the bulk band structure. SOC does not have a strong influence on electronic structure of (111) slab. From our data we deduced quantitative parameters and the type of the superconducting state in SnAs. More specifically,

(i) we confirmed the type I superconductivity in SnAs by features of the specific heat in non-zero magnetic fields, and by the relationship between the estimated quantitative superconducting parameters;

(ii) we found that the temperature dependences of critical magnetic field, specific heat jump, and superconducting energy gap are consistent with the conventional weakcoupling BCS model. Our data shows no signature of the unconventional superconductivity, and the superconducting state in SnAs is likely to have the $s$-type symmetry;

(iii) using two independent techniques (specific heat and Andreev reflection spectroscopy) we determined the superconducting energy gap value $\Delta(0)=0.6 \mathrm{meV}$ - from specific heat data, and $\Delta(0)=0.53 \pm 0.04 \mathrm{meV}$ - from Andreev reflection spectroscopy. These values also satisfy the weak coupling BCS relationship $2 \Delta(0) / T_{c}=3.52$.

Table I summaries our obtained values of the SnAs parameters and compare them with earlier data.

\section{ACKNOWLEDGEMENTS}

Authors thank for valuable discussions E.Z. Kuchinskii and M.V. Sadovskii.

This work was performed using equipment of the LPI Shared Facility Center and the resource center "Physical methods of surface investigation" (PMSI) of the Research park of Saint Petersburg State University.

VMP acknowledges RFBR grant \#16-29-03330. PIB, KAD, AVS, KSP, AVM, ASU, AYT, SYG works were supported within the state assignment of the Ministry of Science and Higher Education of the Russian Federation (project No. 0023-2019-0005).

AGR acknowledge St. Petersburg State University for research Grant No. 15.61.202.2015.

IAN, AAS and NSP aknowledge RFBR grants \#17-0200015 and \#19-32-50001, the Program No. 12 of Fundamental Research of the Presidium of RAS. NSP work was also supported in part by the President of Russia grant for young scientists No. MK-1683.2019.2.

\footnotetext{
* bezpi@sci.lebedev.ru
} 
[1] L.-D. Zhao, S.-H. Lo, Y. Zhang, H. Sun, G. Tan, C. Uher, C. Wolverton, V. P. Dravid, and M. G. Kanatzidis, Nature 508, 373 (2014).

[2] T. H. Hsieh, H. Lin, J. Liu, W. Duan, A. Bansil, and L. Fu, Nature Communications 3, 982 EP (2012), article.

[3] J. Xia, X.-Z. Li, X. Huang, N. Mao, D.-D. Zhu, L. Wang, H. Xu, and X.-M. Meng, Nanoscale 8, 2063 (2016).

[4] Y. Sun, Z. Zhong, T. Shirakawa, C. Franchini, D. Li, Y. Li, S. Yunoki, and X.-Q. Chen, Phys. Rev. B 88, 235122 (2013)

[5] M. Kamitani, M. S. Bahramy, T. Nakajima, C. Terakura, D. Hashizume, T. Arima, and Y. Tokura, Phys. Rev. Lett. 119, 207001 (2017)

[6] Z. Wang, J. Wang, Y. Zang, Q. Zhang, J.-A. Shi, T. Jiang, Y. Gong, C.-L. Song, S.-H. Ji, L.-L. Wang, L. Gu, K. He, W. Duan, X. Ma, X. Chen, and Q.-K. Xue, Advanced Materials 27, 4150 (2015).

[7] Y. Tanaka, Z. Ren, T. Sato, K. Nakayama, S. Souma, T. Takahashi, K. Segawa, and Y. Ando, Nature Physics 8, 800 (2012).

[8] Y. Tanaka, T. Shoman, K. Nakayama, S. Souma, T. Sato, T. Takahashi, M. Novak, K. Segawa, and Y. Ando, Phys. Rev. B 88, 235126 (2013)

[9] L. Fu, Phys. Rev. Lett. 106, 106802 (2011).

[10] S. Sasaki, Z. Ren, A. A. Taskin, K. Segawa, L. Fu, and Y. Ando, Phys. Rev. Lett. 109, 217004 (2012).

[11] M. Sato and Y Y Ando, Reports on Progress in Physics 80, 076501 (2017).

[12] T. Sato, Y. Tanaka, K. Nakayama, S. Souma, T. Takahashi, S. Sasaki, Z. Ren, A. A. Taskin, K. Segawa, and Y. Ando, Phys. Rev. Lett. 110, 206804 (2013).

[13] S. Geller and G. W. Hull, Phys. Rev. Lett. 13, 127 (1964).

[14] Y. Wang, H. Sato, Y. Toda, S. Ueda, H. Hiramatsu, and H. Hosono, Chemistry of Materials 26, 7209 (2014)

[15] H. M. Tütüncü and G. P. Srivastava, Solid State Communications 221, 24 (2015).

[16] P. V. Sreenivasa Reddy, V. Kanchana, T. E. Millichamp, G. Vaitheeswaran, and S. B. Dugdale, Physica B: Condensed Matter 505, 33 (2017).

[17] G. B. Demishev, S. S. Kabalkina, T. N. Kolobyanina, T. I. Dyuzheva, and V. G. Losev, High Pressure Research 1, 325 (1989)

[18] I. Hase, K. Yasutomi, T. Yanagisawa, K. Odagiri, and T. Nishio, Physica C: Superconductivity and its Applications 527, 85 (2016).

[19] P. I. Bezotosnyi, K. A. Dmitrieva, S. Y. Gavrilkin, K. S. Pervakov, A. Y. Tsvetkov, V. P. Martovitski, A. G. Rybkin, and V. M. Vilkov, O. Yu.and Pudalov, JETP Letters 106, 514 (2017).

[20] Y. G. Ponomarev, N. B. Brandt, C. S. Khi, S. V. Tchesnokov, E. B. Tsokur, A. V. Yarygin, K. T. Yusupov, B. A. Aminov, M. A. Hein, G. Müller, H. Piel, D. Wehler, V. Z. Kresin, K. Rosner, K. Winzer, and T. Wolf, Phys. Rev. B 52, 1352 (1995).

[21] T. E. Kuzmicheva, S. A. Kuzmichev, M. G. Mikheev, Y. G. Ponomarev, S. N. Tchesnokov, V. M. Pudalov, E. P. Khlybov, and N. D. Zhigadlo, Phys. Usp. 57, 819 (2014).

[22] M. Octavio, W. J. Skocpol, and M. Tinkham, Phys. Rev. B 17, 159 (1978).

[23] P. Blaha, K. Schwarz, G. K. H. Madsen, D. Kvasnicka, J. Luitz, R. Laskowski, F. Tran, and L. D. Marks, An Augmented Plane Wave + Local Orbitals Program for Calculating Crystal Properties
Techn. Universitt Wien, Austria (2018).

[24] J. P. Perdew, K. Burke, and M. Ernzerhof, Phys. Rev. Lett. 77, 3865 (1996)

[25] D. J. Singh and L. Nordstrom, Planewaves, Pseudopotentials, and the LAPW Method (Springer US, 2006).

[26] M. Uruma, A. Sekiyama, H. Fujiwara, M. Yano, H. Fujita, S. Imada, T. Muro, I. A. Nekrasov, Y. Maeno, and S. Suga, arXiv e-prints , arXiv:0711.2160 (2007), arXiv:0711.2160 [cond-mat.str-el]

[27] A. Damascelli, Z. Hussain, and Z.-X. Shen, Rev. Mod. Phys. 75, 473 (2003).

[28] R. A. Hein and R. L. Falge, Phys. Rev. 123, 407 (1961).

[29] L. L. Zhao, S. Lausberg, H. Kim, M. A. Tanatar, M. Brando, R. Prozorov, and E. Morosan, Phys. Rev. B 85, 214526 (2012).

[30] S. Yonezawa and Y. Maeno, Phys. Rev. B 72, 180504 (2005).

[31] S. Sun, K. Liu, and H. Lei, Journal of Physics: Condensed Matter 28, 085701 (2016).

[32] Y. Yamaguchi, S. Waki, and K. Mitsugi, Journal of the Physical Society of Japan 56, 419 (1987).

[33] V. K. Anand, A. D. Hillier, D. T. Adroja, A. M. Strydom, H. Michor, K. A. McEwen, and B. D. Rainford, Phys. Rev. B 83, 064522 (2011).

[34] V. H. Tran, Z. Bukowski, P. Wisniewski, L. M. Tran, and A. J. Zaleski, Journal of Physics: Condensed Matter 25, 155701 (2013).

[35] F. Bouquet, Y. Wang, R. A. Fisher, D. G. Hinks, J. D. Jorgensen, A. Junod, and N. E. Phillips, Europhys. Lett. 56, 856 (2001).

[36] A. Muratov, A. Sadakov, S. Gavrilkin, A. Prishchepa, G. Epifanova, D. Chareev, and V. Pudalov, Physica B: Condensed Matter 536, 785 (2018).

[37] E. Kuchinskii and M. Sadovskii, Physica C: Superconductivity and its Applications 470, S418 (2010 proceedings of the 9th International Conference on Materials and Mechanisms of Superconductivity.

[38] A. D. Misener, Proceedings of the Royal Society of London A: Mathematical, Physical and Engineering Sciences 174, 262 (1940).

[39] D. C. Rorer, H. Meyer, and R. C. Richardson, Zeitschrift für Naturforschung A 18, 130 (1963).

[40] T. P. Orlando, E. J. McNiff, S. Foner, and M. R. Beasley, Phys. Rev. B 19, 4545 (1979).

[41] R. Kümmel, U. Gunsenheimer, and R. Nicolsky, Phys. Rev. B 42, 3992 (1990).

[42] T. Klapwijk, G. Blonder, and M. Tinkham, Physica B+C 109-110, 1657 (1982).

[43] T. E. Kuzmicheva, A. V. Muratov, S. A. Kuzmichev, A. V. Sadakov, Y. A. Aleshchenko, V. A. Vlasenko, V. P. Martovitsky, K. S. Pervakov, Y. F. Eltsev, and V. M. Pudalov, Physics-Uspekhi 60, 419 (2017).

[44] M. Abdel-Hafiez, P. J. Pereira, S. A. Kuzmichev, T. E. Kuzmicheva, V. M. Pudalov, L. Harnagea, A. A. Kordyuk, A. V. Silhanek, V. V. Moshchalkov, B. Shen, H.-H. Wen, A. N. Vasiliev, and X.-J. Chen, Phys. Rev. B 90, 054524 (2014).

[45] M. Abdel-Hafiez, Y. Zhao, Z. Huang, C.-W. Cho, C. H. Wong, A. Hassen, M. Ohkuma, Y.-W. Fang, B.J. Pan, Z.-A. Ren, A. Sadakov, A. Usoltsev, V. Pudalov, M. Mito, R. Lortz, C. Krellner, and W. Yang, Phys. Rev. B 97, 134508 (2018). 